\author{
Military Technical College \\ Kobry El-Kobbah, \\ Cairo, Egypt.
}

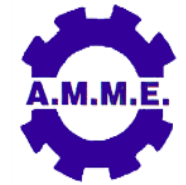

$18^{\text {th }}$ International Conference on Applied Mechanics and Mechanical Engineering.

\title{
DESIGN OF FUZZY CONTROL FOR UNCERTAIN UNDERACTUATED MECHANICAL SYSTEMS
}

\author{
M. M. Emarah ${ }^{*}$
}

\begin{abstract}
This study presents a fuzzy control strategy of underactuated mechanical systems with parametric uncertainties. Underactuated mechanical systems have fewer control inputs than degrees of freedom and arise in applications, such as space and undersea robots, mobile robots, walking, brachiating, and gymnastic robots. The Lagrangian dynamics of these systems may contain feed forward non-linear ties, non-minimum phase zero dynamics, nonholonomic constraints, and other properties that place this class of systems at the forefront of research in nonlinear control. A complete understanding of the control of these systems is therefore lacking. First, this paper represents a brief introduction to the underactuated mechanical systems, the causes of their underactuation and parameters uncertain. Then it deals with the analysis and design of fuzzy controller for one of the application of uncertain underactuated mechanical systems; single flexible link robot. The parameters uncertainties of the flexible robot are presented as bounded variation in payload mass and rotational inertia of the link.
\end{abstract}

\section{KEY WORDS}

Underactuated mechanical systems, Parametric uncertainties, Single flexible link robot, Fuzzy control.

* Lecturer, the High Institute of Engineering, City of Science and Culture, 6th of October City, Giza, Egypt. 


\section{NOMENCLATURE}

$\phi(\mathrm{x}) \quad$ The model shape of the beam.

$\delta(\mathrm{t}) \quad$ The generalized coordinates (generalized modal forces).

$\mathrm{C}(q, \dot{q}) \quad$ The Carioles and centrifugal forces matrix.

CoM Center of Mass of the flexible link.

D Damping matrix of the flexible link.

E Total energy of the system.

EI Beam's rigidity.

F(q) Non-square matrix of external forces.

G(q) The gravity matrix.

$J \quad$ The total inertia of the flexible beam.

$\mathrm{J}_{\mathrm{o}} \quad$ The actuator inertia.

$\mathrm{J}_{\mathrm{p}} \quad$ Payload inertia including $40 \%$ variation.

$\mathrm{J}_{\mathrm{po}} \quad$ Payload inertia.

$\mathrm{K} \quad$ Spring matrix of the flexible link.

$\mathrm{K}_{\mathrm{e}} \quad$ The kinetic energy.

L The Lagrangian function.

$L \quad$ The link length.

M Total inertia matrix of the flexible link including $40 \%$ variation.

M(q) The inertia matrix.

$\mathrm{M}_{\mathrm{L}} \quad$ Inertia matrix of the flexible link.

$m_{p} \quad$ Payload mass including $40 \%$ variation.

$m_{p o} \quad$ Payload mass.

$\mathrm{n}_{\mathrm{e}} \quad$ Number of selected modes.

$q \quad$ The vector of generalized coordinate.

$T \quad$ Torque input to the link actuator.

$\mathrm{u} \quad$ The input generalized force.

$V \quad$ The potential energy.

$W(x, t) \quad$ Bending deformation.

$\theta(t) \quad$ Position of the CoM to the link.

$\theta_{c}(t) \quad$ Position of the tangent to the link base.

$\theta_{t}(t) \quad$ Position of a line pointing to the beam tip.

$\omega \quad$ The natural (unforced) frequency.

$\Delta \mathrm{J}_{\mathrm{p}} \quad$ Variation in payload inertia of the flexible link.

$\Delta \mathrm{M}_{\mathrm{L}} \quad$ The perturbation inertia matrix of the flexible link.

$\Delta \mathrm{m}_{\mathrm{p}} \quad$ Variation in payload mass of the flexible link.

$\rho \quad$ Uniform density.

\section{INTRODUCTION}

An underactuated mechanical system is the mechanical system that has fewer independent control inputs than the degree of freedom of the system to be controlled. This class of systems has been the subject of many scientific researches followed by their broad applications in different branches of knowledge. Research of underactuated mechanical systems began from two decades ago when control of nonholonomic mechanical systems were of great interest by scientists "Spong" [1997] and "Saber"[2001], and they generated interesting control problems to which traditional control theory was not relevant. Examples of such systems include mobile 
robot, spacecraft, underwater vehicle, surface vessel, helicopter, space robot, and underactuated manipulator (such as flexible manipulators).

The problem of underactuated mechanical systems is to control the system by using fewer independent actuators than degree of freedom. In order to understand this problem, it is necessary to understand the cause of underactuation. Some of those causes are; a system may become underactuated when one or more of its actuators fail to work properly and it must complete its task under this satiation. Another reason makes the system may become underactuated when it is specifically designed to be so, or when the designer keeps one or more of the actuators available inoperative during a task. Underactuation may be an essential property of the system, like freefloating space satellite equipped with a conventional robot manipulator. Also, it imposed artificially to create complex low-order nonlinear systems for the purpose of gaining insight into controlling high-order underactuated mechanical systems, (e.g. the cart-pole system, the beam and ball system, the Acrobot, the Pendubot) [1].

Control of underactuated mechanical systems is now one of the most important research fields in control. The efforts of scientists and engineers led to the important research on nonlinear control theory, optimal control, adaptive control, and other control theories. Finally, the research of underactuated mechanical systems becomes essential now days. In a practical control problem, many constraints have to be handled in order to design controllers which operate in a real environment. First, an appropriate model for the system must be fond. If the model gives an accurate representation of physical phenomena then a satisfactory control law design could be achieve, which give an observed behavior of the real controlled system is conform to the desired results. Generally, to obtain a model operated in real environment, it is necessary to approximate or neglect some phenomena, or to choose some main parameters. A direct consequence is that the derived model is affected by some uncertainties. To find a control operating in a real environment, uncertainties have to be appropriately described and their effects considered in the control law design.

Fuzzy logic was applied in many research on uncertainty modeling and controlled, it is generally agreed that "Zadeh's" seminal paper in 1965 has the pioneering role as the starting point of the modern concept of uncertainty. In his paper, "Zadeh" [1965] introduced a theory whose objects are fuzzy sets of which the boundaries are not precise and the membership to a fuzzy set is not a matter of confirmation, but rather a matter of degree [2]. Most of the researches of underactuated mechanical systems have less considered practical issues such as the robustness against parameter uncertainty, un-modeled dynamics and other reasons of uncertainties [3-7].

\section{MODELING OF UNDERACTUATED MECHANICAL SYSTEMS}

Generally, the mathematical models for underactuated mechanical systems are highly nonlinear and heavily coupled ones, the mathematical models of such systems usually consist of a set of linear or nonlinear differential/difference equations derived by using some form of approximation and simulation. The dynamic models of underactuated mechanical systems may depend not only on their structure but also on their internal characteristics. 


\section{Equations of Motion}

The equations of motion of underactuated mechanical system used in most literatures were obtained from the simple Lagrangian mechanical system [1] expressed as below;

$\frac{d}{d t} \frac{\partial L}{\partial \dot{q}}-\frac{\partial L}{\partial q}=F(q) u$

where:

$\mathrm{L}=K_{e}-V$, is the Lagrangian parameter.

$K_{e}$, is the kinetic energy.

$V$, is the potential energy of the system.

$q \in R^{n}$ is the vector of generalized coordinate.

$u \in R^{m}$ is the input generalized forces.

$F(q) \in R^{n \times m}$ is a non-square matrix of external forces.

In the vector form, equation (1) is written as:

$$
M(q) \ddot{q}+C(q, \dot{q}) \dot{q}+G(q)=F(q) u
$$

where,

$M(q)$ is the inertia matrix which is uniformly positive definite matrix.

$\mathrm{C}(\mathrm{q}, \dot{q})$ contains the Carioles and centrifugal forces.

$G(q)$ contains the effects of gravity.

The total energy of the system $E$, for conservative systems, gives:

$$
E=K_{e}+V
$$

The simple Lagrangian mechanical system expressed by equations (2) can represent both fully-actuated mechanical system and underactuated mechanical system according to the column rank of $F(q)$. The simple Lagrangian mechanical system is called a fully-actuated mechanical system if $m=\operatorname{rank}(F(q))=n$, or is called an underactuated mechanical system if $m<n$.

\section{Case Study: Single Flexible Link Robot}

The link flexibility of a robotic manipulator must be considered in modeling and control when the manipulator is of large dimension or lightweight. Large manipulators play important roles in many applications, such as construction automation, environmental applications, and space engineering. Lightweight arms have great potential for design of high-performance industrial robotic manipulators since they allow high speed operation and low energy consumption. Most efforts in the modeling and control of flexible manipulators have been on the understanding of a single flexible link shown in (Fig.1). Due to the complexity involved with link deformation, however, establishing the exact dynamic model even for one link flexible manipulators is unrealistic, and certain simplifying approximations about the link 
deformation have to be made. The study of single flexible link based on finite element method is introduced in [8].

The Flexible link is modeled using Euler-Bernoulli Beam theory in many other references like in [8, and 9]. The control strategy based on collocated and noncollocated feedback linearization is considered as one of the important technique in other research [10]. The intelligent control including fuzzy control is used in control the trajectory tracking problem of the flexible link robot is presented in $[8,11$, and 12].

The significant physical properties of the beam are defined as: Length $L$, Uniform density $\rho$, Young modulus $E$, Cross-section inertia I, The actuator inertia $J_{0}$, Payload mass $m_{p}$ and inertia $J_{p}$. The specification of the link is shown in Table1. The beam dynamic model is developed based on the following assumptions:

1- The flexible link is a slender beam.

2- The beam undergoes small deformations of pure bending type in the plane of motion (no torsion or compression).

3- Bending deformation $\mathrm{W}(\mathrm{x}, \mathrm{t})$, with $\mathrm{x} \in[0, \mathrm{~L}]$, is directed along the $y$ direction (no shear).

4- Rotational inertia of beam sections is neglected (Timoshenko theory) as well as the isoperimetric constraint ('extension' of beam neutral axis is negligible).

Some other relevant angular variables are selected to be $\theta(t), \theta_{c}(t)$ and $\theta_{t}(t)$ with definition:

- Position of the $\operatorname{CoM}$ is $\theta(\mathrm{t})$.

- Position of the tangent to the link base is $\theta_{c}(t)$.

- Position of a line pointing to the beam tip $\theta_{\mathrm{t}}(\mathrm{t})$.

The model of one flexible link is derived using the Lagrangian is formed from kinetic and elastic potential energy of the beam. Using Hamilton principle and calculus of variations, the bending deformation $\mathrm{W}(\mathrm{x}, \mathrm{t})$ and angle $\theta(\mathrm{t})$ are satisfy the linear differential equations,

$$
\begin{aligned}
& E I W^{\prime \prime \prime}(x, t)+\rho(\ddot{W}(x, t)+x \ddot{\theta}(t))=0 \\
& T(t)-J \ddot{\theta}(t)=0
\end{aligned}
$$

where, $T=$ torque input, and $J$ the total inertia of the flexible beam. The dynamic model is described as,

$$
\begin{aligned}
& J \ddot{\theta}=T \\
& \ddot{\delta}_{i}+2 \zeta_{i} \omega_{i} \dot{\delta}_{i}+\omega_{i}^{2} \delta_{i}=\left(\frac{\phi_{i}^{\prime}(0)}{J_{L}}\right) T \\
& \zeta_{i} \in[0,1)
\end{aligned}
$$

Equation (6) is written in the classical mass-spring-damper form, as follows;

$$
M \ddot{q}+D \dot{q}+K q=B T
$$

where the generalized coordinates are: 
$q=\left(\theta \delta_{1} \ldots \delta_{n e}\right)^{T}$

$M=\left[\begin{array}{ll}J & 0 \\ 0 & I\end{array}\right]$,

$D=\left[\begin{array}{cc}0 & 0 \\ 0 & 2 Z \Omega\end{array}\right]$,

$K=\left[\begin{array}{cc}0 & 0 \\ 0 & \Omega^{2}\end{array}\right]$,

$B=\left[\begin{array}{l}1 \\ \phi^{\prime}\end{array}\right]$,

$\Omega=\operatorname{diag}\left\{\omega_{1}, \ldots \ldots ., \omega_{n e}\right\}$

$Z=\operatorname{diag}\left\{\zeta_{1}, \ldots \ldots, \zeta_{n e}\right\}$

and

$\phi^{\prime}=\frac{1}{J_{L}}\left(\phi_{1}^{\prime}(0), \ldots \ldots . ., \phi_{n e^{\prime}}(0)\right)^{T}$.

The total inertia of the single link flexible arm is given by:

$\mathrm{J}=\mathrm{J}_{\mathrm{o}}+\left(\rho \mathrm{L}^{3}\right) / 3+\mathrm{J}_{\mathrm{p}}+\mathrm{m}_{\mathrm{p}} L^{2}$

where

$m_{p}=m_{p o}+\Delta m_{p}$

$I_{p}=J_{p o}+\Delta J_{p}$

The dynamic system including the variation of payload mass and rotational inertia can be designed as:

$\left(M_{l}+\Delta M_{L}\right) \ddot{q}+D q \dot{q}+K q=B T$

where,

$M_{L}=\left[\begin{array}{cc}l_{2} & 0 \\ 0 & I\end{array}\right]$

$\Delta M_{L}=\left[\begin{array}{cc}\Delta J_{2} & 0 \\ 0 & I\end{array}\right]$

$I_{2}=J_{0}+\frac{\rho L^{3}}{3}+J_{p o}+m_{p o} L^{2}$ 
$\Delta J_{2}=\Delta J_{p}+\Delta m_{p} L^{2}$

and $\Delta M_{2}$ is the perturbation inertia matrix contains the uncertain parameters of mass payload and rotational inertia. The variation of the payload mass and inertia are selected to be bounded and scalar perturbations.

$\theta_{t}=\theta+\sum_{i=1}^{n e} \frac{\phi_{i}(L)}{L} \delta_{i}$

Tip angle $\theta_{t}$ as defined in(9) is selected to be the control output as it is affected by the vibratory motion $\delta_{i}(t)$, so, it is important to be controlled to follow the desired trajectory with minimum vibration effect. The tip deflections $\phi_{i}(\mathrm{~L})$ are function of payload mass and inertia, so they are change according to the variation of payload mass and inertia

\section{CONTROLLER DESIGN}

Recently, one of the most important control techniques is the intelligent control; which is defined as a combination of control theory, operation research and artificial intelligence. Fuzzy controllers are expert control system that uses common sense rules and natural language statement. In real control systems several tasks are done by human, those tasks must be performed based on the evaluation of the measured data according to a set of rules which the human expert has learned from experience or training.

Fuzzy logic control has many advantages that make it very powerful when applied to highly nonlinear and/ or ill defined systems; as it is suitable for both linear and nonlinear systems, it is more robust than the classical control, and it can deal with systems which is ill mathematical defined or have no mathematical models at all.

Fuzzy control system can be used as closed-loop controllers. In this case the fuzzy system measures the outputs of the process and takes control actions on the process continuously. The fuzzy controller uses input fuzzy sets to generate by an inference scheme, which is based on a knowledge base of control force to be applied on the system [3, and 4].

This establishes a fuzzy logic controller to control nonlinear vibration of a single flexible link robot. In feedback loop of the control system, a fuzzy logic controller is used to provide control torques for the system and used to generate the joint torques and to enhance the performance of the system in vibration process with different suggested trajectories focus on follow the trajectory with minimum possible tip vibration.

The closed loop control system with fuzzy PD control is designed, the position error, the change of the error and the output controller fuzzy membership functions, three sets of gains multiply the inputs and the output $K_{p}, K_{d}$ and $K_{u}$ respectively. Considering the variation of payload mass and inertia with the desired of best performance (the link follows the desired input reference with minimum error), the sets of gains are estimated. 
Although the performance of the hub and tip angle (rigid motion) to the applied unit step is accepted, the two flexible modes show unstable response (flexible motion). The changing in the gains shows no better response to the flexible modes. This is because of the "control energy" is spilled over on the flexible modes and caused it to be excited. This instability could be corrected by adding a PI or lag controller that reduced spill over and the oscillation are approximately eliminated. For simplicity, the control output from the PD fuzzy controller taken as the change of control torque (du/dt) and integrate the control output to be the applied control torque to the model as shown in Fig. 2. This led to that the overall control system.

\section{SIMULATION RESULTS}

The dynamic model with parameter uncertainties of a single link flexible manipulator is described in Section 2. The uncertainty in parameters is described as a variation in the link payload mass and rotation inertia. The payload mass $m_{p}$ and rotational inertia $J_{p}$ are selected to have bounded variation of (40\%) from the defined link specifications shown in Table 1. during the operation [5]. The MatLab and Simulink package is used to simulate the performance of a single flexible link

An investigation of the flexible deflection of the link with the variation of payload mass and rotational inertia for the first five modes using Bisection Method is shown in Fig. 3. The results show small deviations for the first five modes, the variation for the first two modes show the maximum values compare to the higher modes (the fixable deflection variation by $8.97 \%$ in Fig. 3 [a], the natural frequency change by $13.74 \%$ in Fig. 3 [b] and the tip model shape function deviation by $32.19 \%$ in Fig. 3 [c]). The analysis shows that the tip deflection for two and four modes for extreme values of mass payload and inertia give approximately the same response as shown in Fig.3 [d]. For the given results above and as the increasing in the number of modes leads to complicated model to solve, the single flexible link is modeled up to the second mode only.

The closed loop system with fuzzy logic controller of nonlinear dynamic model of single link flexible robot is design and simulated as shown in Fig. 2. In this study, fuzzy logic controller has two inputs and one output: the position error (e), change in the error (de) and the change of torque (du). Input and output fuzzy members functions are symmetric and triangle member functions were used in membership functions. For the fuzzy controller, 7 fuzzy sets defined over each variable, the number of rules equal to $7^{2}=49$ rules and are shown in Table 2.

The fuzzy controller rules are in the form:

\section{If $e$ is $e_{i}$ and de is $D e_{i}$ then du is $D u_{i}$}

where, $e_{i}=$ linguistic term of error.

$$
\begin{aligned}
& \mathrm{De}_{i}=\text { linguistic term of change in error. } \\
& \mathrm{Du}_{i}=\text { linguistic term of change in torque. }
\end{aligned}
$$

The scaling factors; which scale the real system values into the normalized one are $\mathrm{K}_{p}, \mathrm{~K}_{d}$ and $\mathrm{K}_{u}$ such that,

$$
\text { Inputs; } \boldsymbol{e}_{\boldsymbol{i}}=\boldsymbol{K}_{\boldsymbol{p}} \boldsymbol{e}
$$




$$
\begin{aligned}
& D e_{i}=K_{d} d e \\
& \text { Output; } D u_{i}=K_{u} d u=K_{u}\left(K_{p} e+K_{d} d e\right)
\end{aligned}
$$

The control system forced the single flexible link robot to follow the given input (step input) with tip deflection with maximum overshoot of [18\% - 21\%] at [5.2-6.25 sec] and steady state error less than 0.001 for different payload mass and inertia, as shown in Fig. 4.

The control system is applied to the link to follow several desired trajectories with minimum vibration. The desired trajectory (1) is designed as a series of step and ramp as show in Fig.5 [a]. Another desired trajectory (2) is approximated to be parabolic position profile in Fig.5 [c]. Both trajectories are designed using the "Signal Builder" in MatLab and Simulink package

The simulation results show in Fig. $5[b, d]$, the link follows the desired trajectory with approximation zero tip vibration. The fuzzy controller shows a high performance on the single flexible link robot.

\section{CONCLUSION}

In this paper, a brief acknowledge of underactuated mechanical systems and parameters uncertainties of the real system are introduced. The single flexible link robot is considered as underactuated mechanical system. It is selected to modeled and control with parameters uncertainties. The uncertainties are represented as variation of payload mass and rotational inertia. The dynamic model including the variation of payload mass and inertia included in the inertia matrix. With bounded variation of $40 \%$ in payload mass, slight deviation of the link flexible deflections parameters as shown in the simulated results and for the first two flexible modes. This study proposes a fuzzy control strategy which required to overcome the flexible effect of the link and forced the tip to trace the desired input trajectory with min. error and deal with the variation in payload mass and rotational inertia. The control strategy with a set of gains schedule shows acceptable performance of the single flexible link robot with minimum tip vibration.

\section{REFERENCES}

[1] Aneke, "Control of Underactuated Mechanical Systems", A Dissertation Submitted To The Graduate School In Partial Fulfilment Of The Requirements for the degree of Doctor Of Philosophy, Technische Universiteit Eindhoven, 2003.

[2] Jantzen. "Foundations of Fuzzy Control", John Wiley \& Sons, Ltd, 2007.

[3] Celikyilmaz and Türksen "Modeling Uncertainty with Fuzzy Logic", SpringerVerlag Berlin Heidelberg, 2009.

[4] Song and Hedrick "Dynamic Surface Control of Uncertain Nonlinear Systems", Springer-Verlag Berlin Heidelberg,2011

[5] Farooq, Gu, El-Hawary, Luo, Asad. "Takagi Sugeno Fuzzy Controlled for Uncertain Single Link Manipulator", the Ninth International Conference on Advanced Engineering Computing and Applications in Sciences, 2015 
[6] Mamani, Becedas, Feliu. "Sliding Mode Tracking Control of a Very Light Weight Single-Link Flexible Robot Robust to Payload Changes and Motor Friction", Journal of Vibration and Control, Sage, 2011.

[7] Qiu, Wang, Yuan, Rao. "A Control Strategy Combining Adaptive Fuzzy Control and Dynamic Equilibrium State Theory for Acrobot with System Uncertainty", Information Technology Journal 13 (6), 2014.

[8] A. M. Abdullahi, Z. Mohamed and M. Muhammad. "A Pd-Type Fuzzy Logic Control Approach For Vibration Control Of A Single-Link Flexible Manipulator", International Journal of Research in Engineering and Science, 2013.

[9] W. N. White, M. Foss, and X. Guo. "A Direct Lyapunov Approach for a Class of Underactuated Mechanical Systems", Proceedings of the 2006 American Control Conference Minneapolis, Minnesota, USA, 2006.

[10] Jee-Hwan Ryu, Dong-Soo Kwon, and Blake Hannaford, "Control of a Flexible Manipulator With Noncollocated Feedback: Time-Domain Passivity Approach", IEEE Transactions On Robotics, VOL. 20, NO. 4, 2004.

[11] T. M. MANSOUR and S. KAZI. "Fuzzy Logic Modified Proportional-IntegralDerivative (MPID) Control for Flexible Manipulator", International Journal of Circuits, Systems And Signal Processing, 2013

[12] A. M. Abdullahi, Z. Mohamed, M. Muhammad, A.A. Bature. "Vibration Control Comparison Of A Single Link Flexible Manipulator Between Fuzzy Logic Control And Pole Placement Control", International Journal of Scientific \& Technology Research, Volume 2, ISSUE 12, 2013 


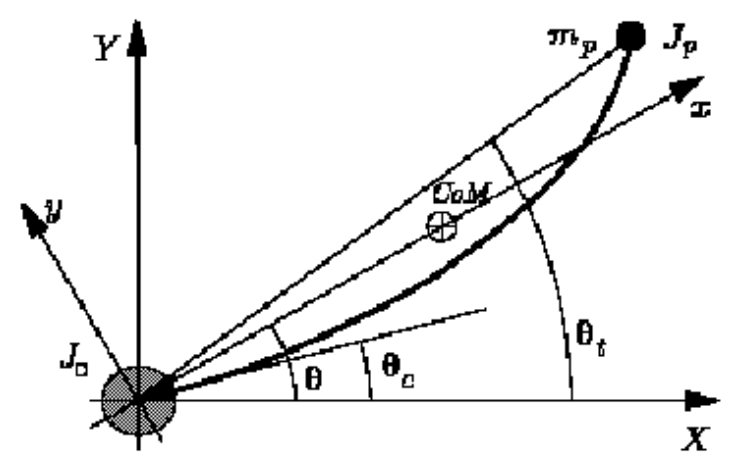

Fig.1. Single flexible link robot.

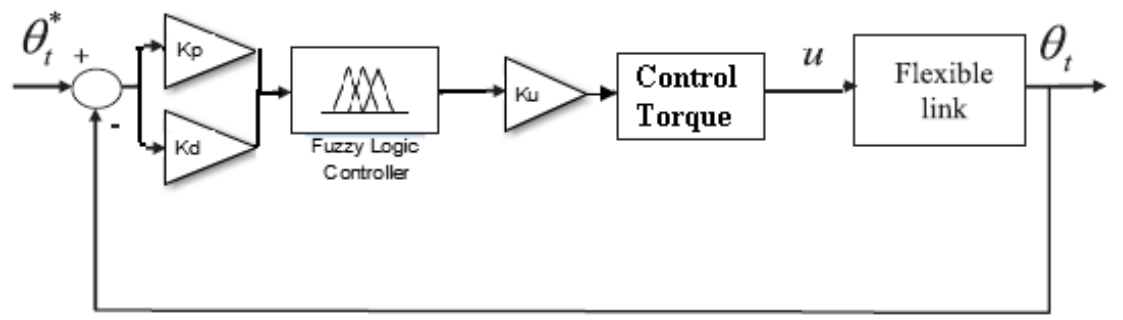

Fig. 2. The control system of a single flexible link robot with fuzzy logic controller.

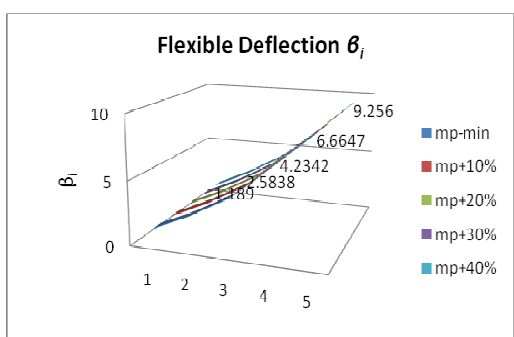

[a]

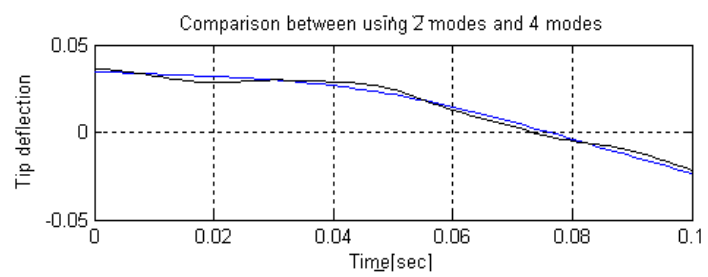

Tip deflection using 2 and 4 modes with normal payload mass.

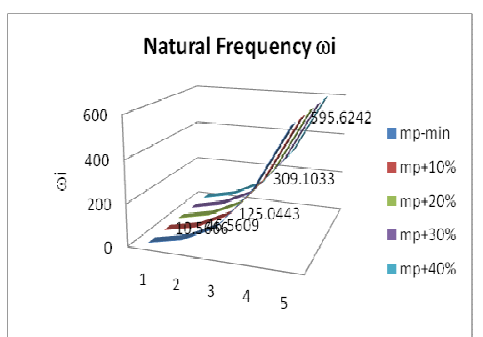

[b]

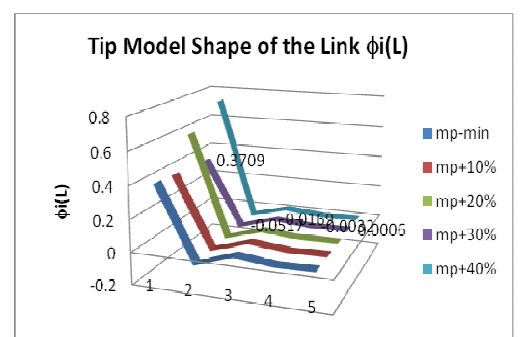

[c]

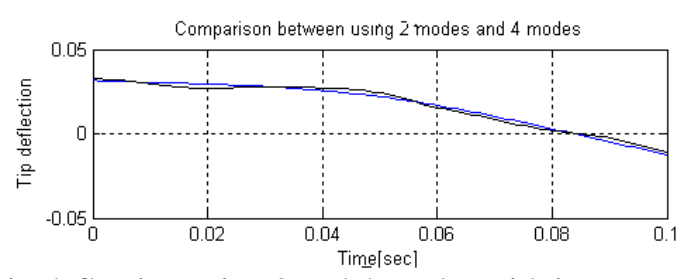

Tip deflection using 2 and 4 modes with increase of $40 \%$ in payload mass.

[d]

Fig. 3. The flexible deflection results with payload mass variation. 


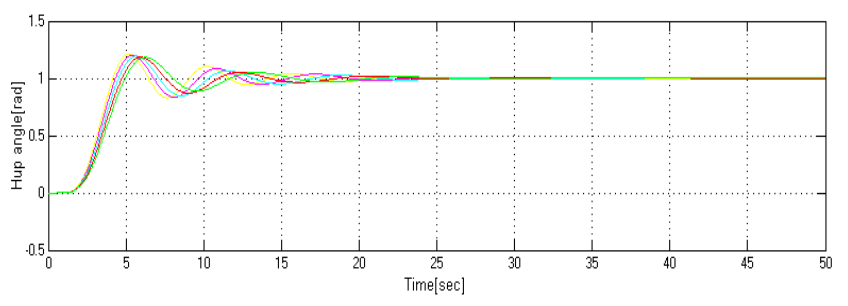

[a] Hup angle [rad]

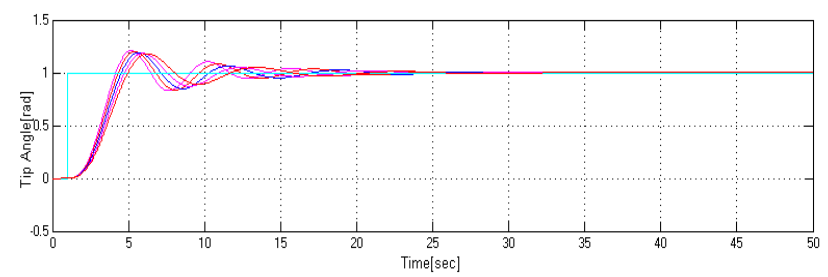

[b] Tip angle [rad]

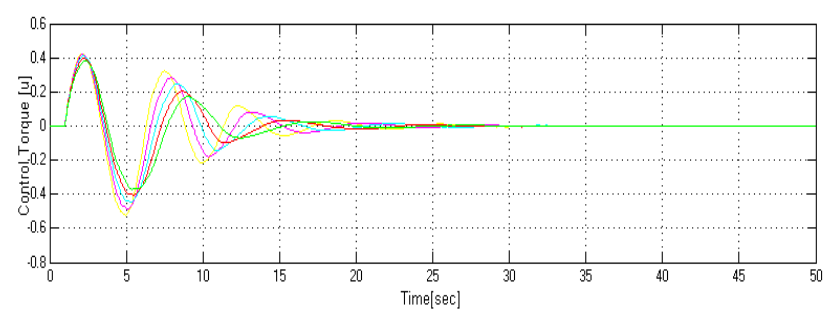

[c] Control Torque [J]

Fig. 4. The Simulation results of one flexible link subjected to a unit step input for different payload mass.

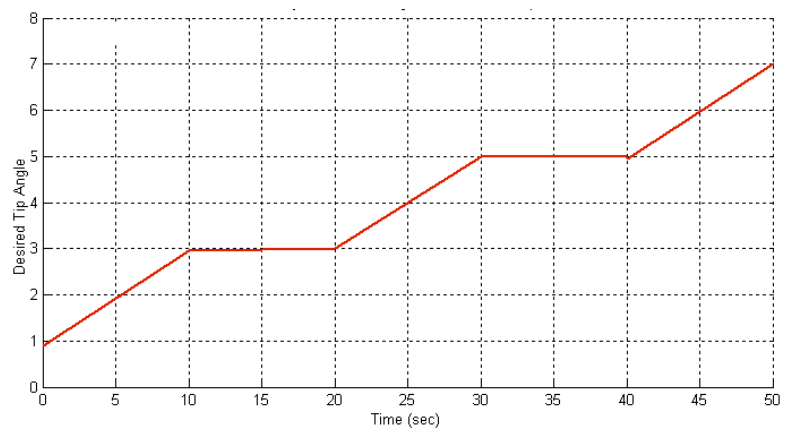

[a] The desired trajectory (1)

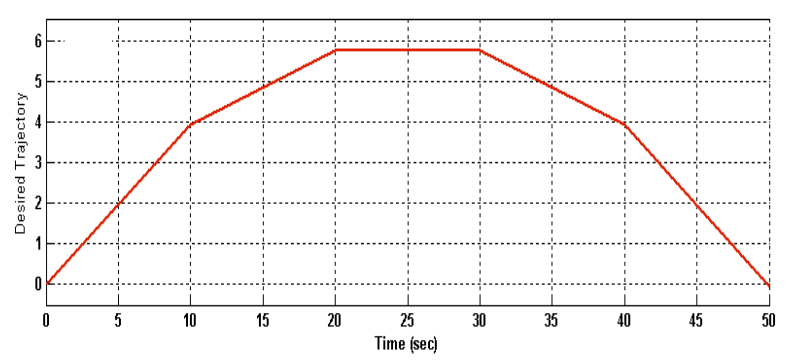

[c] The desired trajectory (2)

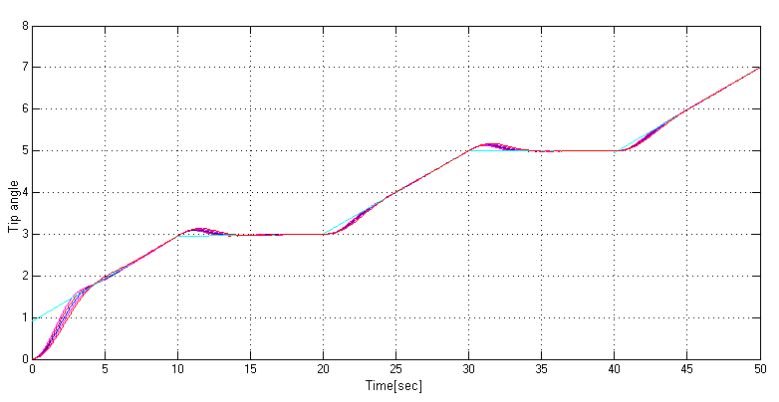

[b] Tip angle of the desired trajectory (1).

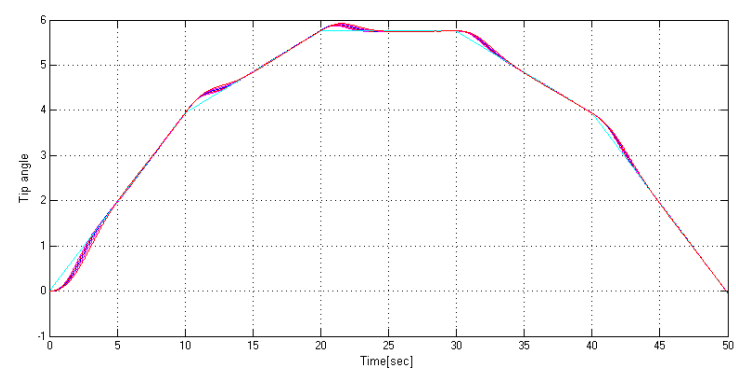

[d] Tip angle of the desired trajectory (2).

Fig. 5. The Simulation results of one flexible link subjected to trajectory (1) and (2) for different payload mass. 
Table 1. Specification of the single flexible link robot.

\begin{tabular}{|l|ll|}
\hline The actuator inertia $\mathrm{J}_{\mathrm{o}}$ & $\mathrm{J}_{\mathrm{o}}=0.016$ & {$\left[\mathrm{~kg} \cdot \mathrm{m}^{2}\right]$} \\
\hline The link length is $\mathrm{L}$ & $\mathrm{L}=1.1938$ & {$[\mathrm{~m}]$} \\
\hline Uniform density $\rho$ & $\rho=0.2457$ & {$[\mathrm{~kg} / \mathrm{m}]$} \\
\hline Beam's rigidity $\mathrm{El}$ & $\mathrm{EI}=11.85$ & {$\left[\mathrm{~N} . \mathrm{m}^{2}\right]$} \\
\hline Payload mass $\mathrm{m}_{\mathrm{po}}$ & $\mathrm{m}_{\mathrm{po}}=0.5867$ & {$[\mathrm{~kg}]$} \\
\hline Max. increase in payload mass $\Delta \mathrm{m}_{\mathrm{po}}$ & $\Delta \mathrm{m}_{\mathrm{po}}=0.2347$ & {$[\mathrm{~kg}]$} \\
\hline Payload inertia $\mathrm{J}_{\mathrm{po}}$ & $\mathrm{J}_{\mathrm{po}}=0.2787$ & {$\left[\mathrm{~kg} \cdot \mathrm{m}^{2}\right]$} \\
\hline Max. increase in payload inertia $\Delta \mathrm{J}_{\mathrm{po}}$ & $\Delta \mathrm{J}_{\mathrm{p}}=0.1115$ & {$\left[\mathrm{~kg} \cdot \mathrm{m}^{2}\right]$} \\
\hline
\end{tabular}

Table 2. Fuzzy logic rules.

\begin{tabular}{|c|ccccccc|}
\hline du & \multicolumn{7}{c|}{ de } \\
\hline e & NB & NM & NS & ZO & PS & PM & PB \\
\cline { 3 - 6 } NB & PB & PB & PB & PM & PM & PS & ZO \\
NM & PB & PB & PM & PM & PS & ZO & NS \\
NS & PB & PB & PS & PS & ZO & NS & NM \\
ZO & PB & PM & PS & ZO & NS & NM & NB \\
PS & PM & PS & ZO & NS & NS & NB & NB \\
PM & PS & ZO & NS & NM & NM & NB & NB \\
PB & ZO & NS & NM & NM & NB & NB & NB \\
\hline
\end{tabular}

